\title{
Hybrid PAPR Reduction Scheme for Universal Filter Multi-Carrier Modulation in Next Generation Wireless Systems
}

\author{
Pooja Rani, Silki Baghla and Himanshu Monga* \\ Department of ECE, JCDM College of Engineering, India \\ *Corresponding author: Himanshu Monga, Department of ECE, Director of JCDM College of Engineering, Sirsa, India \\ Submission: 監 November 10, 2017; Published: 監 January 08, 2018
}

\begin{abstract}
Universal filter multi carrier (UFMC) is one of the promising multi carrier modulation techniques for next generation wireless communication systems. UFMC seems to be most attractive because it provides better sub carrier separation like FBMC (Filer Bank Multi Carrier) and less complexity like OFDM (Orthogonal Frequency Division Multiplexing). But this technique suffers from limitation of higher Peak to Average Power Ratio (PAPR). In this paper a Hybrid PAPR reduction technique SC- UFMC have been proposed using SLM (Selective Mapping) and Clipping. The performance of proposed technique is evaluated for various design parameters including filter length, FFT size and Bits per sub carrier. The simulation results show that hybrid technique provides better PAPR reduction as compared with conventional SLM and clipping techniques.
\end{abstract}

Keywords: UFMC; OFDM; FBMC; PAPR; SLM

\section{Introduction}

Orthogonal Frequency Division Multiplexing (OFDM) is most popular multi-carrier modulation technique which is being used in 4th generation wireless communication [1]. But in last few years, number of users and demand for higher data rates has been increased exponentially so, next generation wireless communication systems must be able to deal with large number of users and provide a much higher data transmission rate using less complex system. In order to serve all these requirements, various new multi carrier modulation techniques like Filter bank Multi carrier (FBMC), Universal Filter Multi Carrier (UFMC) and Generalized Frequency Division Multiplexing (GFDM) have been introduced $[2,3]$.

In FBMC, each subcarrier is individually filtered and provides robustness against inter-carrier interference (ICI) effects [4]. However, FBMC systems utilize filters, whose length is multiple times of samples per multi-carrier symbol resulting in increased complexity of the system. Universal Filtered Multi-Carrier (UFMC) is a novel multi-carrier modulation technique, which combines the features of FBMC and OFDM. UFMC filters groups of subcarriers instead of per sub carrier like FBMC or complete signal in single shot like OFDM. This allows reducing the filter length considerably as compared to FBMC. So, it is less complex like OFDM and provides better sub carrier separation like FBMC $[5,6]$. The main drawback of all these multicarrier modulation techniques is high Peak to Average Power Ratio $[7,8]$.
In this paper a novel hybrid PAPR reduction technique SCUFMC for UFMC systems has been proposed. This technique is implemented by using SLM and Clipping PAPR reduction techniques. It is observed that this Hybrid technique provides better results when compared with the individual performances of SLM and Clipping techniques.

\section{UFMC waveform generation}

For next generation wireless communication system a new waveform is required which should achieve the asynchronous reception and transmission, non-orthogonal waveforms for better spectral efficiency and low latency. UFMC has been introduced as a new waveform design representing a generalization of this principle targeting to collect the advantages while avoiding the disadvantages of other modulation techniques $[9,10]$. UFMC is the method which combines the advantages of orthogonality of OFDM and concept of filter bank in FBMC. Instead of filtering each carrier like in FBMC, block of carriers called sub-bands are filtered. Each sub-band contains a number of carriers and filter length will depend upon the width of sub-band [11]. Figure 1 shows process of transmission and reception in UFMC system. Here, the complex symbols generated from the modulator (QPSK or QAM) are applied to serial to parallel converter resulting in a block of streams and fed as input to their respective IFFT . The length of $\mathrm{N}$ point IFFT output is converted back to serial per block and that output will be filtered with a pulse shaping filter of length $\mathrm{L}$. 
The generated output can be given as:

$$
x=\sum_{p}^{N_{R B}} F_{p} V_{p} X_{k}^{i}
$$

The data stream $\mathrm{x}$ is converted to $\mathrm{N}$ RB disjoint blocks and each sub-block is applied to individual $\mathrm{N}$ point IFFT represented by matrix ' $V p$ '. The output of IFFT is converted to serial form and applied to respective filter represented by matrix ' $\mathrm{Fp}^{\prime}$ in eq.1. The complete process is shown in Figure 1. The signal output of each block can be expressed as [12]:

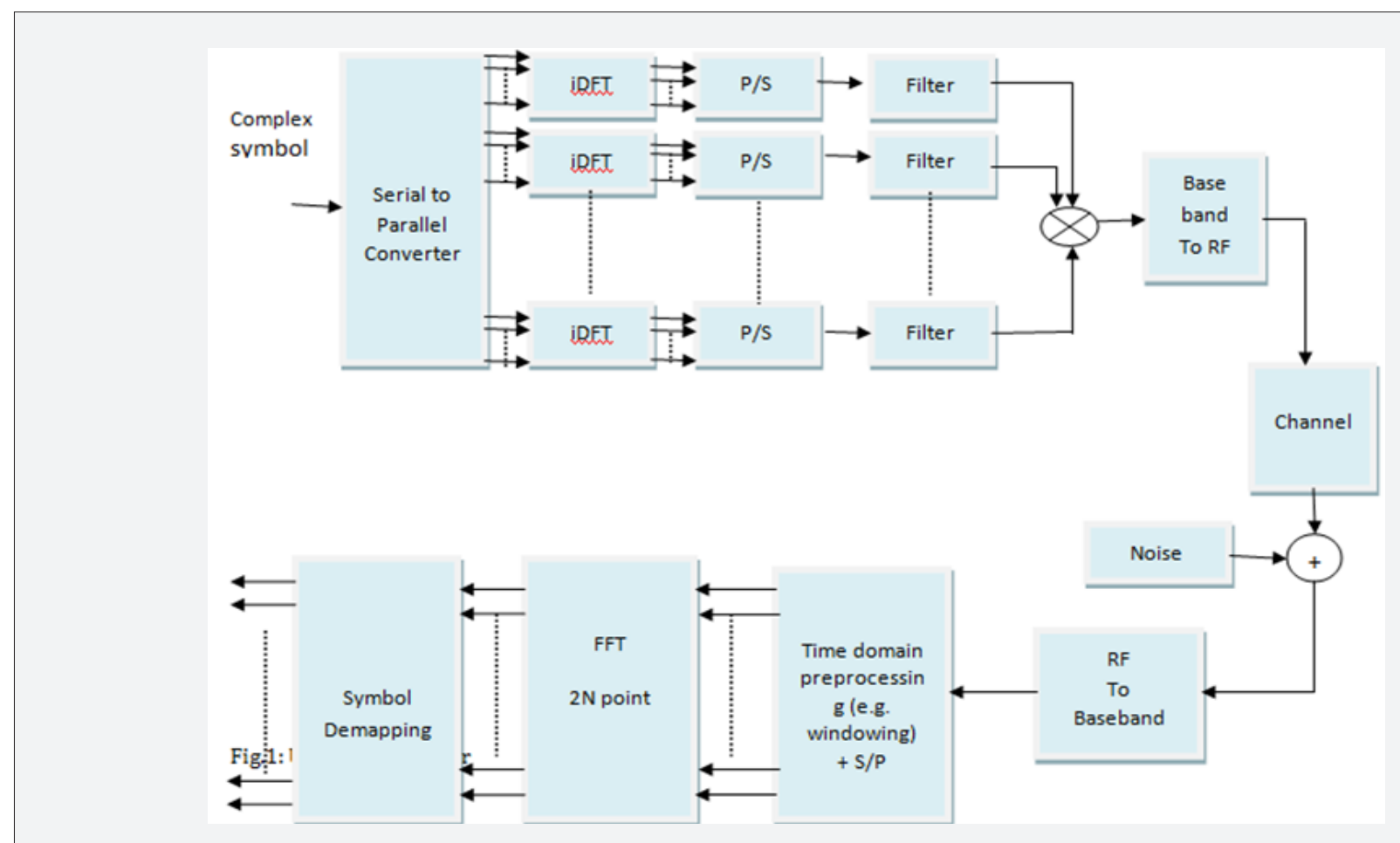

Figure 1: Process of transmission and reception in UFMC system.

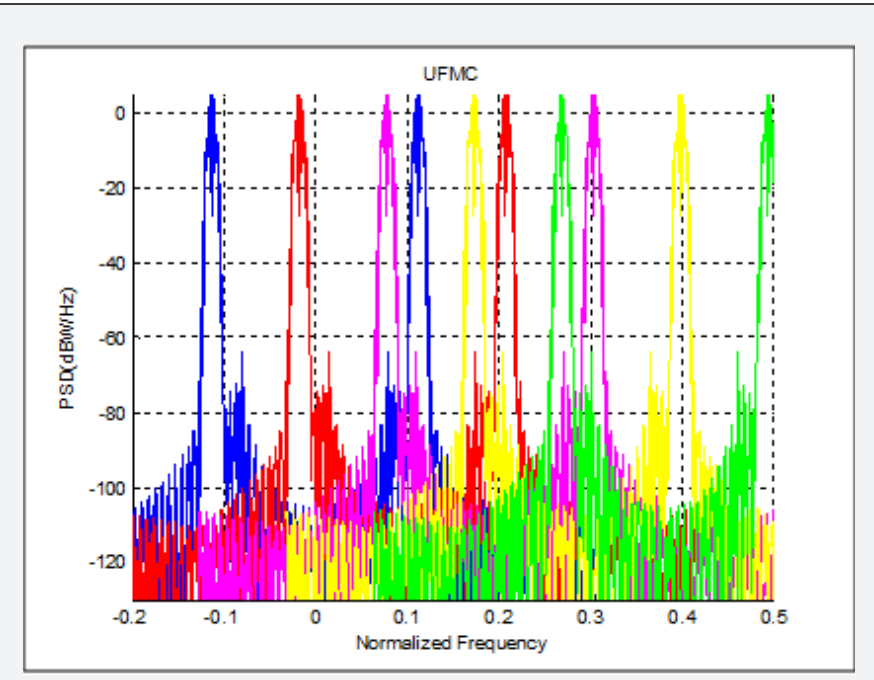

Figure 2: UFMC waveform.

$$
\begin{aligned}
& \bar{F}=\left[F_{1}, F_{2}, F_{3}, \ldots \ldots . F_{N_{R B}}\right] \\
& \overline{V_{p}}=\operatorname{diag}\left(V_{1}, V_{2}, V_{3}, \ldots . . V_{N_{R B}}\right)
\end{aligned}
$$

$$
\begin{gathered}
\overline{X_{k}^{i}}=\left[X_{k, 1}^{i^{T}}, X_{k, 2}^{i^{T}}, X_{k, 3}^{i^{T}}, \ldots \ldots X_{k, N_{R B}}^{i^{T}}\right]^{T} \\
x=\bar{F} \overline{V_{p}} X_{k}^{i}
\end{gathered}
$$

At receiver, after passing through RF-link section the signal is applied to time domain pre-processing window to suppress interfer $\urcorner$ ence. After windowing, the signal will be converted into ' $2 \mathrm{~N}$ ' parallel streams; here ' $\mathrm{N}$ ' is the number of subcarriers. The demodulated signal is sent to the demapper, which is a demodulator to retrieve the data bits from the received symbols. The generated waveform is shown in Figure 2.

The block-wise filtering provides flexibility to the system and may be used to avoid the main drawbacks of FBMC. UFMC supports short bursts data transmission, as well as operation in fragmented bands. The filter provides protection against intersymbol interference (ISI), as well as robustness for supporting multiple access users which are not perfectly time-aligned. Due to the possibility to reduce guard bands, and to avoid need of CP, UFMC is spectrally more efficient than CP-OFDM [13]. The receiver processing can still be similar to CP-OFDM, single-tap per-subcarrier frequency domain equalizers can be used which equalize the joint 
impact of the radio channel and the respective sub band-filter. This leads to similar complexity order as CP-OFDM. So, it is thereby clear that UFMC provides advantages of both OFDM and FBMC system.

\section{Peak to average power ratio}

UFMC has numerous advantages over other modulation techniques, but it also suffers from high Peak to Average Power Ratio (PAPR).The PAPR is the relation between the maximum power of a sample in a given transmitted symbol divided by the average power of that symbol. PAPR occurs when in a multicarrier system the different sub-carriers are out of phase with each other. There are a large number of independently modulated subcarriers in multicarrier system which are different with respect to each other at different phase values. When all the subcarriers achieve the maximum value simultaneously, this will cause the output envelope to suddenly increase which causes a 'peak' in the output, and when they are added up coherently for transmission purpose give a large peak value which is very large as compared to average value of the sample. The ratio of the peak to average power value is termed as Peak-to-Average Power Ratio [14]. The mathematical valuation of PAPR is defined in equation (6).

$$
\operatorname{PAPR}=\left(\frac{\max \left\{|x[n]|^{2}\right\}}{E\left\{|x[n]|^{2}\right\}}\right)
$$

Where, $|x[n]|$ is the amplitude of $\mathrm{x}[\mathrm{n}]$ and $\mathrm{E}$ denote the expectation of the signal.

This higher PAPR causes saturation in power amplifier which produces inter modulation products among sub bands and also increases out of band radiation (OOB).

\section{Conventional PAPR Reduction Techniques}

There are various PAPR reduction techniques which can be used to improve performance of UFMC systems. Some of them are:

\section{Selective mapping (SLM) technique}

In SLM, from the original data block several candidate data blocks are generated and all the data blocks are having the same information. After this a phase rotation is applied to each block and passed from it's respective iDFT and a block with minimum PAPR is selected for transmission [15].

\section{Companding}

Companding is an easy and less complex method of PAPR reduction, the basic idea is to expand the small signal in transmitter section and compression is carried out at receiver side. In this technique, we enlarge the small signals while compressing the large signals to increase the immunity of small signals from noise. This compression is carried out at the transmitter end, after the output is taken from IFFT block. There are two types of companders: $\mu$-law and A-law companders [16].

\section{Partial transmit sequence}

In PTS original data block is partitioned into $\mathrm{N}$ disjoint sub blocks. The subcarriers in each sub block are rotated by the same phase factor such the PAPR of the combination can be minimized. PTS scheme reduces PAPR with some additional complexity and it also affects spectral efficiency of the system because side information is also required to be transmitted. It does not produce any distortion in system [17].

\section{Clipping and filtering}

This is one of the simplest techniques for PAPR reduction. The principle is to define a clipping level for data transmission above which the input signal is clipped off and peaks of signal are reduced [18]. Let, there is a signal $\mathrm{y}[\mathrm{n}]$ which is to be transmitted and $y_{c}[n]$ is its clipped version which can be denoted as:

$$
y_{c}[n]=\left\{\begin{array}{l}
-A y[n] \leq-A \\
y[n]|y[n]|<A \\
A y[n] \geq A
\end{array}\right\}
$$

Where, A is the clipping level. After clipping out of band radiations are produced this can be reduced by using filtering after clipping.

\section{Tone reservation}

In this scheme, some subcarriers are reserved within the transmitted bandwidth and appropriate value is assigned to these reserved tones [19]. These reserved subcarriers don't carry any data information, are only used for reducing PAPR.

\section{Active constellation extension (ACE)}

In ACE, at each block, some of the outer signal constellation points are extended towards outside of the constellation such that the PAPR of the resulting block is reduced. It is transparent to receiver. There is no loss of data rate and no side information is required.

\section{Proposed Hybrid Scheme}

In this work, we have proposed a novel Hybrid technique for PAPR reduction in UFMC by using SLM and Clipping. Unlike existing SLM and Clipping schemes for OFDM systems, the proposed method exploits the nature of sub-block data transmission of UFMC. In UFMC data is generated in form of sub blocks which are group of sub carriers. Advantages of SLM and Clipping have been exploited in this Hybrid technique. In Clipping some distortion is produced but SLM there is not any distortion while on the other hand SLM produces data rate loss but in Clipping there is not any data rate loss. So, in this work we have combined these two schemes so that we can exploit advantages of both schemes. Both techniques are having advantage that power of system is not increase. Hence, we can decrease PAPR with the same power which is used by the system when there is not any PAPR reduction technique is applied.

\section{Hybrid (SC-UFMC) PAPR reduction technique}

The basic block diagram of Hybrid technique is shown in Figure 3. Waveform generated by UFMC modulator is given to serial to parallel converter where several candidate blocks are generated from original data block; this is done to find a block 
with minimum PAPR for transmission. After generating candidate blocks a phase rotation is applied to each block and applied to IFFT of their respective. Because of the varying assignment of data to the transmit signal, it is called selective mapping. The core is to choose a particular signal which is having desired properties out of $\mathrm{n}$ signals representing the same information. Finally, we select a block with minimum PAPR, the signal generated by this selector is applied to clipper for removing the higher peaks. For this a perticular thershold value is defined above which all the signal is clipped of so that peak to average power ratio can be reduced.

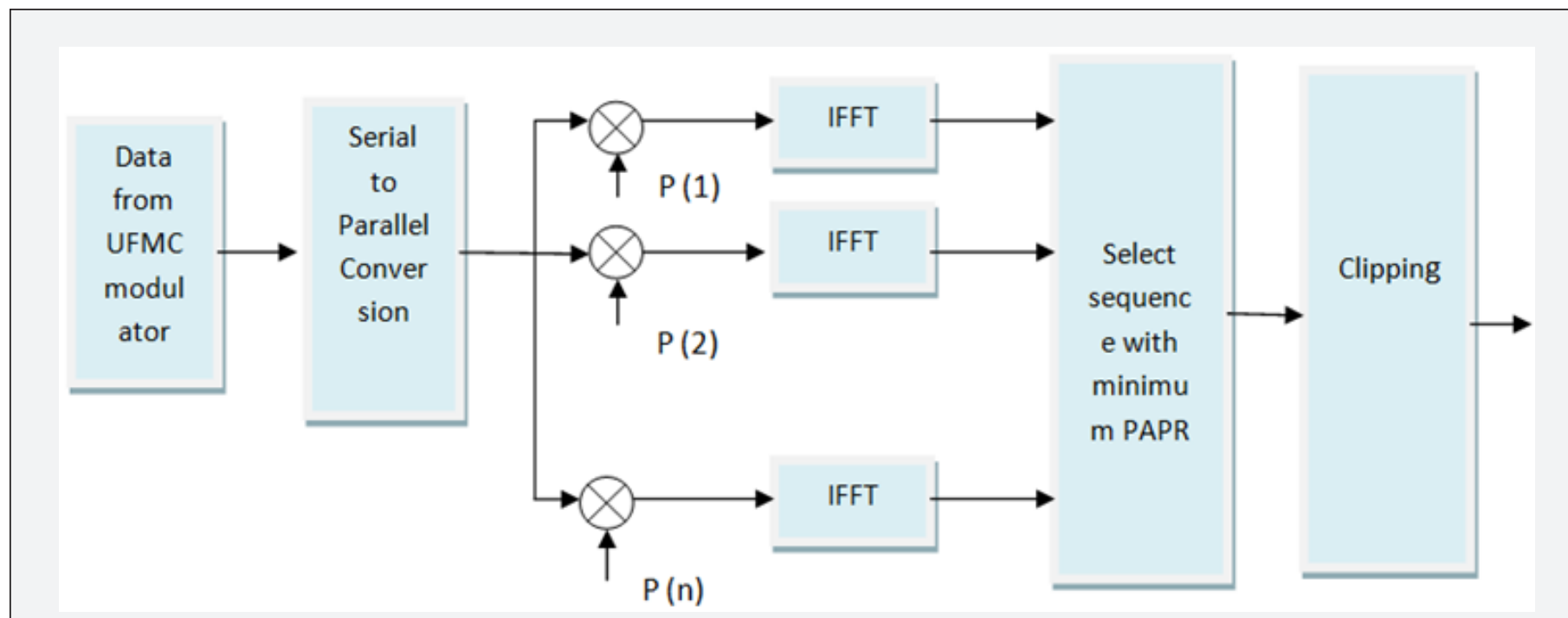

Figure 3: Block diagram of hybrid (SC-UFMC) technique

The signal genrated by UFMC is given in equation 6 and after applying a phase rotation the signal become as given in equation (7).

$$
x=\sum_{p=1}^{N_{R B}} F_{p} V_{p} X_{k}^{i} p(n)
$$

Where, $p(n)$ denotes the phase rotation of the signal, after this signal after FFT is applied to clipper. Let the threshold for the signal is $\mathrm{A}$. Then the final signal will be:

$$
x_{c}=\left\{\begin{array}{l}
x,|x|<A \\
A,|x|>A
\end{array}\right\}
$$

Hence, by using this technique we selected a block with minimum PAPR and then a clipper circuit is used to clip of the peaks of that block so that we can reduce peak to average power ratio of signal to a great extent.

\section{Simulation Setup and Results}

\section{Simulation setup}

Table 1: Simulation setup.

\begin{tabular}{|c|c|}
\hline Parameter & Values \\
\hline FFT Size & $2,04,81,02,45,12,256$ \\
\hline Sub Band Size & 20 \\
\hline Number of Sub bands & 10 \\
\hline Modulation order & QAM $(4,16,64)$ \\
\hline Bits per Sub Carrier & $2,4,6$ \\
\hline Filter Length & $43,63,83$ \\
\hline
\end{tabular}

Table 1 provides the simulation set up to evaluate the performance of proposed PAPR reduction technique. The proposed technique is also compared with three other techniques as original (UFMC), with Selective mapping (S-UFMC), with Clipping (C-UFMC) and Hybrid (SC-UFMC) to analyze the effectiveness in PAPR reduction. The performance of proposed method is evaluated and compared on the basis of variation in FFT size, Bits Per sub carrier, filter length and modulation order.

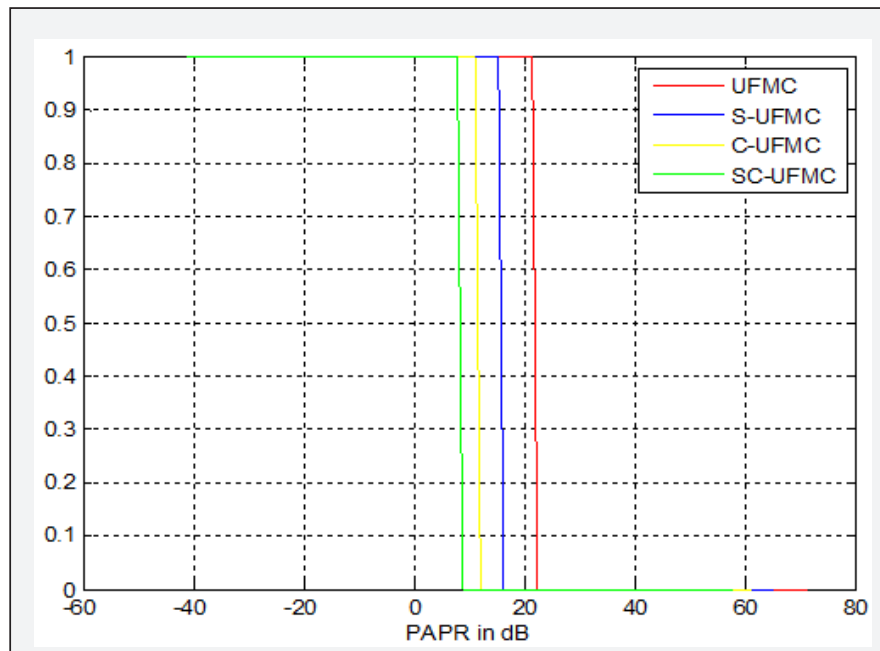

Figure 4: PAPR reduction of different schemes.

In Figure 4 Peak to Average Power Ratio of UFMC, SLM scheme (S-UFMC), Clipping scheme (C-UFMC) and Hybrid scheme (SCUFMC) with FFT Size 1024, Filter length 43, Bits per sub carrier 2 and modulation order 4 are shown. It can be concluded that the proposed hybrid schemes have improved PAPR reduction performance as compared with the two conventional schemes for PAPR reduction. 


\section{Performance evaluation of SC-UFMC with variation in design parameters}

This section presents performance evaluation of SC-UFMC with variation in design parameters (FFT Size, Bits per sub carrier and Filter length).

\section{Variation in FFT Size}

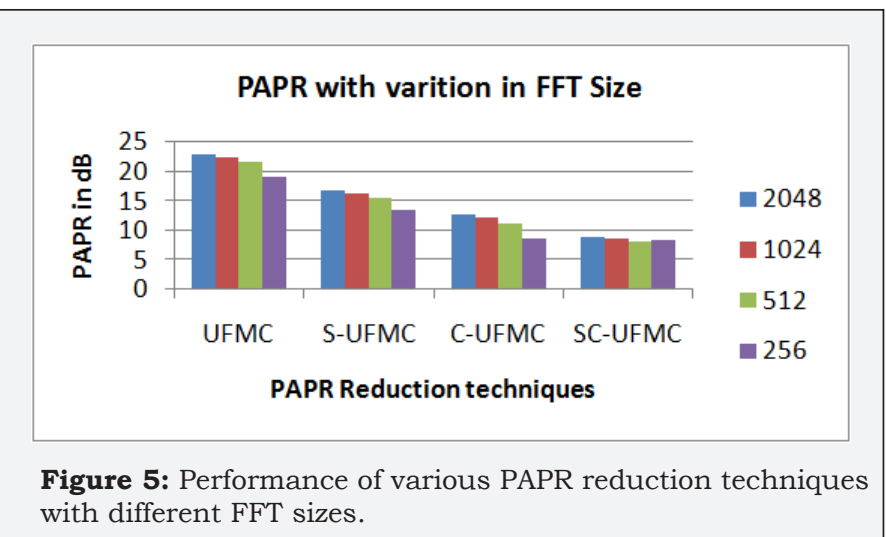

Figure 5 shows the PAPR of UFMC signal and performance of three PAPR reduction techniques at FFT size 2048. It can be observed that SC-UFMC is providing better PAPR reduction than other two techniques.

As shown in Figure 4 C-UFMC shows better performance than S-UFMC, so effectiveness of SC-UFMC is analyzed with different values of design parameters with respect to C-UFMC. Table 2 is showing that SC-UFMC performs more effectively with larger FFT size.

Table 2: Effectiveness with variation in FFT size.

\begin{tabular}{|c|c|}
\hline FFT Size & \% Effectiveness of SC-UFMC \\
\hline 256 & $4.23 \%$ \\
\hline 512 & $27 \%$ \\
\hline 1024 & $28.15 \%$ \\
\hline 2048 & $30.14 \%$ \\
\hline
\end{tabular}

Variation in Bits per sub carrier

PAPR with variation in Bits per sub carrier

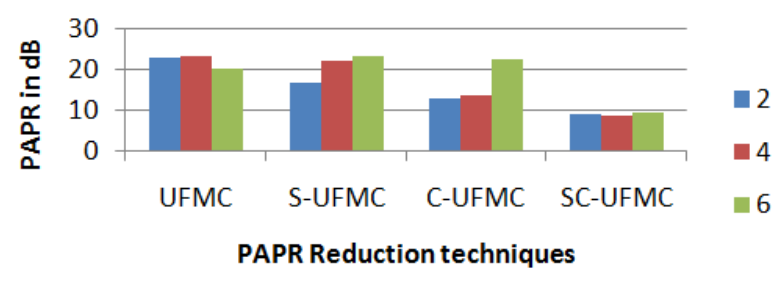

Figure 6: Performance of various PAPR reduction techniques with different bits per sub carrier.

Performance of various PAPR reduction techniques with 2 Bits per sub carrier is shown in Figure 6 from here it can be observed that proposed scheme is showing better results than conventional schemes. All the schemes are showing different values when we change any of the design parameters as shown in Figure 6 here we are changing bits per sub carriers.

Table 3: Effectiveness with variation in bits per sub carrier.

\begin{tabular}{|c|c|}
\hline Bits per Sub carrier & \%Effectiveness of SC-UFMC \\
\hline 2 & $30.14 \%$ \\
\hline 4 & $37.62 \%$ \\
\hline 6 & $58.60 \%$ \\
\hline
\end{tabular}

As shown in Table 3 with increase in Bits per sub carrier, SCUFMC is being more effective and at 6 bits per sub carrier it is having maximum PAPR reduction.

\section{Variation in filter length}

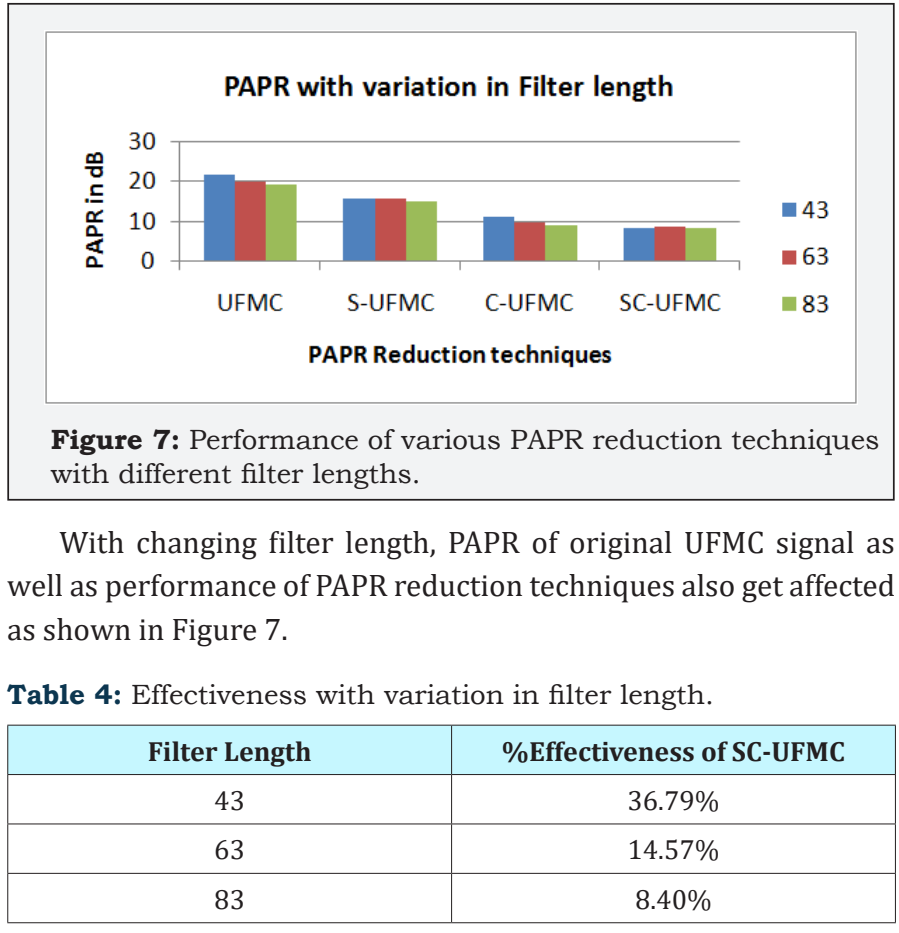

It can be observed from Table 4 that at higher filter length proposed scheme performed less effectively and a better PAPR reduction can be obtained with lower filter length.

\section{Conclusion}

In this paper, a novel hybrid scheme SC-UFMC for PAPR reduction of UFMC signals is proposed. Simulation results and analysis shows that the Hybrid scheme is an efficient PAPR reduction method for UFMC systems, and it can provide better PAPR reduction performance than the conventional SLM and Clipping schemes. Further, effect of various design parameters on PAPR reduction in SC-UFMC has been analyzed and it is concluded that proposed technique provide effective PAPR reduction than conventional schemes.

\section{References}

1. 5GNOW (2013) D3.1: 5 G waveform candidate selection. Tech Rep.

2. Rohde, Schwarz (2016) Application Note "5G waveform candidates". 
3. Schaich F, Wild T (2014) Waveform contenders for 5G-OFDM vs. FBMC vs. UFMC. ISCCSP pp. 457-460.

4. Farhang-Boroujeny B (2011) OFDM versus filter bank multicarrier. IEEE Signal Process Mag 28(3): 92-112.

5. Vakilian V, Wild T, Schaich F, Ten Brink S, Frigon JF (2013) Universalfiltered multi-carrier technique for wireless systems beyond LTE. IEEE Globecom Workshops pp. 223-228.

6. Wunder G, Jung P, Kasparick M, Wild T, Schaich F, et al. (2014) 5GNOW: non-orthogonal, asynchronous waveforms for future mobile applications. IEEE Commun Mag 52(2): 97-105.

7. Arun G, Manushree B (2012) An overview: Peak to average power ratio in OFDM system \& its effect. International Journal of Communication and Computer Technologies 1(2).

8. Pooja M, Silki B, Himanshu M (2016) PAPR reduction methods for multicarrier modulation schemes used in next generation wireless networks-A review. International Journal of Broadband Cellular Communication 2(2): 35-44.

9. Chen Y, Schaich F, Wild T (2014) Multiple access and waveforms for 5G: IDMA and universal filtered multi-carrier. IEEE $79^{\text {th }}$ VTC Spring, pp. 1-5.

10. Wild T, Schaich F, Chen Y (2014) 5G Air interface design based on universal filtered (UF-) OFDM. $19^{\text {th }}$ International Conference on Digital Signal Processing, pp. 699-704.

11. An C, Kim B, Ryu HG (2016) Waveform comparison and nonlinearity sensitivities of FBMC, UFMC and W-OFDM systems. $8^{\text {th }}$ International Conference on Networks \& Communications, pp. 83- 90.

12. Pooja R, Silki B, Himanshu M (2017) Effect of transmission parameters on PAPR of universal filter multicarrier modulation systems.

13. Pooja R, Silki B, Himanshu M (2017) Performance evaluation of multicarrier modulation techniques for next generation wireless systems. International Journal of Advances in Computer Science 8(5): 508-511.

14. Tellado J, Cioffi JM (1999) PAR reduction in multicarrier transmission system. Stanford University, USA.

15. Robert WB, Robert FHF, Johannes BH (1996) Reducing the peak to average power ratio of multi carrier modulation by selected mapping. IEEE Electronics Letters 32(22): 2056 - 2057.

16. Wang X, Tjhung TT, Ng CS (1999) Reduction of peak to average power ratio of OFDM system using a companding technique. IEEE Transactions on Broadcasting 25.

17. Muller SH, Huber JB (1997) OFDM with reduced peak-to-average power ratio by optimum combination of partial transmit sequences. IEEE Electronics letters 33(5): 368-369.

18. O'Neill R, Lopes LB (1994) Performance of Amplitude Limited Multitone Signals. In proceedings of IEEE $44^{\text {th }}$ Vehicular Technology Conference 1675-1679.

19. Brian SK, Douglas LJ (2002) A new tone reservation method for complex baseband PAR reduction in OFDM system. ICASSP 3(4): pp. 2321-2324. 